\title{
In memory of Professor Leonid I. Manevitch
}

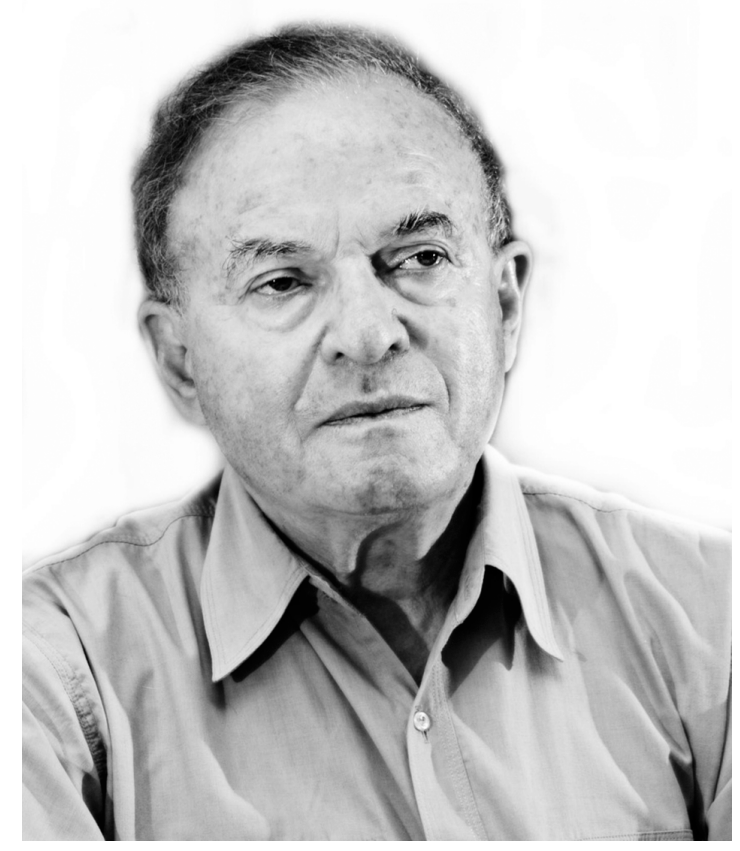

The Russian and World Science has suffered the bereavement. On August 20, 2020, Professor, Doctor of Sciences Leonid Isakovich Manevitch passed away after terminal illness.

Leonid Isakovich Manevitch was born on April 2, 1938 in Mogilev (USSR, currently Belarus). He got higher education at the Faculty of Physics and Mathematics of Dnepropetrovsk State University (DSU) in 1954-1959. After graduating from the DSU, he worked as an aerospace stress engineer and a head of theoretical group in the Yangel Yuzhnoye State Design Office (1959-1964) and at the same time he studied in extramural Doctoral study in DSU. He got the degree of Candidate of Sciences (PhD) for his thesis on stability of shells in 1961 (under supervision by Prof. Yu.A. Shevlyakov). From 1964 till 1976 he worked in DSU as an Associate Professor, and then as a Full Professor at the Department of Applied Theory of Elasticity (led by Academician V.I. Mossakovsky). In 1970 he received the degree of Doctor of Sciences for his thesis devoted to asymptotic and group methods in the mechanics of deformable solids, and the title of Professor was conferred upon him in 1973. Since 1976, after moving to Moscow, he worked as a senior research fellow, and then as a head of the Laboratory of the Polymer Physics and Mechanics of the Institute for Chemical Physics of the USSR Academy of Sciences. Since 1984 he also worked as a Professor at the Department of Polymer Physics at the Moscow Institute of Physics and Technology. L.I. Manevitch's scientific activity was related to many areas of mechanics of deformable media, nonlinear dynamics and condensed matter physics. He obtained important and valuable results in the problems of strength and stability of anisotropic 
and reinforced plates and shells, in the of elasticity theory for anisotropic media, including theory of deformation and destruction of highly anisotropic composites. He has made significant contributions to the theory of nonlinear normal oscillations in essentially nonlinear systems, to nonstationary dynamics of nonlinear oscillatory systems; to molecular dynamics and physics of polymers and composite materials. He developed the theory of structural defects and structural transitions at the atomic level in molecular and polymer crystals, studied the transport and localization of energy in mechanical systems, polymer chains and nanotubes. These works have numerous applications to various fields of mechanics and physics, the theory of vibration protection and nanotechnology. L.I. Manevitch was an active participant of many Russian and international symposia, conferences and congresses. As a guest speaker he repeatedly appeared at seminars of famous universities of the USA, Europe and Israel (Harvard University, Massachusetts Institute of Technology, Brown University, University of Illinois Urbana-Champaign, Delft University of Technology, Universities of Groningen, Ulm, Freiburg, Technion, etc.). His scientific results are presented in 20 monographs (in publishing houses Wiley, Springer, Kluwer, Imperial College Press, Nauka (Russia)) and in more than 350 publications. L. I. Manevitch's boundless enthusiasm and outstanding pedagogical talent allowed him to create a large scientific circle of affiliates and followers. L. I. Manevitch provided great influence on the work and lives of all his students and collaborators, which are employed in the universities and the research centers around the world. We remember his constant attention and support in our study and work. He was a supervisor on 38 candidate $(\mathrm{PhD})$ thesises, and 12 of his disciples became Doctors of Science. Up to last days of his life, L. I. Manevitch was in the head of the Laboratory of Physics and Mechanics of Polymers. This laboratory under his leadership became one of the leading research teams in the field of solid polymer physics and nonlinear dynamics of molecular systems, which actively cooperates with famous research centers from different countries. The colleagues, disciples and friends will be always indebted to the Professor L. I. Manevitch.

Prof. Yuri V Mikhlin, National Technical University, Kharkov, Ukraine Prof. Igor Adrianov, Rheinisch-Westfälische Technische Hochschule Aachen (RWTH Aachen University), Koeln, Germany

Prof. Vladimir Astashev, Mechanical Engineering Research Institute, RAS, Moscow, Russia Prof. Valery Pilipchuk, Wayne State University, Detroit, MI, USA

Prof. Oleg Gendelman, Technion - Israel Institute of Technology, Haifa, Israel

Prof. Julius Kaplunov, School of Computing and Mathematics, Keele University, Keele, UK

Prof. Yuli Starosvetsky, Technion - Israel Institute of Technology, Haifa, Israel

Doctor Valeri Smirnov, Federal Research Center for Chemical Physics, RAS, Moscow, Russia Doctor Margarita Kovaleva, Federal Research Center for Chemical Physics, RAS, Moscow, Russia 